\title{
Many-body theory of pump-probe spectra for highly excited semiconductors
}

\author{
T. J. Inagaki*甘, T. Iida ${ }^{\ddagger}$ and M. Aihara* \\ * Graduate School of Materials Science, Nara Institute of Science and Technology, Ikoma, Nara 630-0101, Japan \\ ¥ Department of Physics, Osaka City University, Sumiyoshi-ku, Osaka 558-8585, Japan
}

(November 13, 2018)

\begin{abstract}
We present a unified theory for pump-probe spectra in highly excited semiconductors, which is applicable throughout the whole density regime including the high-density electron-hole BCS state and the low-density excitonic Bose-Einstein condensate (BEC). The analysis is based on the BCS-like pairing theory combined with the Bethe-Salpeter (BS) equation, which first enables us to incorporate the state-filling effect, the band-gap renormalization and the strong/weak electronhole pair correlations in a unified manner. We show that the electron-hole BCS state is distinctly stabilized by the intense pump-light, and this result strongly suggests that the macroscopic quantum state can be observed under the strong photoexcitation. The calculated spectra considerably deviate from results given by the BCS-like mean field theory and the simple BS equation without electronhole pair correlation especially in the intermediate density states between the electron-hole BCS state and the excitonic BEC state. In particular, we find the sharp stimulated emission and absorption lines which originate from the optical transition accompanied by the collective phase fluctuation mode in the electron-hole BCS state. From the pump-probe spectral viewpoint, we show that this fluctuation mode changes to the exciton mode with decreasing carrier density.
\end{abstract}

PACS numbers: 71.35Lk, 42.65.-k, 42.65.Pc

\section{INTRODUCTION}

The many-body effects of electron-hole systems in semiconductors generated by the intense laser field have long been attractive subjects 1 . and the macroscopic quantum state can easily be controlled by changing the frequency and the intensity of excitation light. Recent developments in the experimental techniques make it possible to observe the remarkable phenomena suggesting the optically induced macroscopic quantum states. In particular, the anomalous exciton transport observed in $\mathrm{Cu}_{2} \mathrm{O}$ (Ref. 3) and $\mathrm{BiI}_{3}$ (Ref. 4 ) are the typical examples of them. These phenomena become more significant with increasing the electron-hole densities, and are in a marked contrast to the simple ballistic exciton propagation or the conventional diffusive exciton transport.

In order to understand the nature of the macroscopic guantum state of electron-hole systems, conventional approaches based either on the weak interacting Boson modella or on the BCS-like mean-field theory This is because the mean interparticle distance in the highly photoexcited system is often of the same order as the radius of the bound electron-hole pairs, and the system is in the intermediate state between the excitonic Bose-Einstein condensate (BEC) and the electron-hole BCS state. The nature of the system is characterized by two kinds of order: the ${ }^{4} \mathrm{He}$-like order associated with the center-of mass motion of electron-hole pairs and the BCS-like order associated with the electron-hole pair formation. In order to investigate the physical properties of the system, we have to incorporate the state-filling effect, which reflects the Pauli principle, and the center-of-mass motion of electron-hole pairs on the same footing.

In our previous work $\mathrm{G}$, we have presented a theory of luminescence spectra for electron-hole systems in highly excited semiconductors; this theory gives the correct description of the luminescence spectra in the crossover regime between the low-density excitonic BEC states and the high-density electron-hole BCS states. The analysis is based on the generalized random-phase approximation combined with the Bethe-Salpeter (BS) equation $\mathbf{3}$. This approach enables us to incorporate the strong electron-hole pair correlation and the quantum fluctuation effect associated with the center-of-mass motion of electron-hole pairs. We have shown that the crossover from the excitonic BEC state to the electron-hole BCS state manifests itself in the luminescence spectra. In particular, it is found that the broad emission band, arising from the pair recombination in the electron-hole BCS state, splits into the $\mathrm{P}$ and $\mathrm{P}_{2}$ emission band\$s, 10 with decreasing the electron-hole density. These results are closely related fo the recent noteworthy experiment for $\mathrm{ZnO}$, in which the room temperature ultraviolet laser emission is observed 11 .

The purpose of the present work is to clarify the properties of the high-density electron-hole systems subject to the intense light by analyzing the pump-probe spectrum. To our knowledge, a many-body theory for absorption spectrum of semiconductors was first presented by Löwenau et al.22; their analysis can be applied both to the lowand high-density states. They solved the BS equation for the interband dielectric function by a numerical matrix 
inversion 13.44, and reproduces a series of excitonic sharp spectral components in the low particle densities. However, the electron-hole pair correlation in the high-density regime is not taken into account, so that the many exciton effect and the BCS-like macroscopic quantum state cannot be incorporated.

In Ref. 15, the effect of pair correlation is taken into account for the pump-probe spectrum by the simple BCSlike mean-field theory at zero temperature. By solving the BCS-like gap equation for electron-hole systems, they found that the electron-hole pair correlations are considerably enhanced by the pump-light intensity ("light enhanced excitons"). Their calculated spectra exhibit the transparent region between the stimulated emission and the absorption components, which is the manifestation of the BCS-like gap formation. Whereas their theory takes into account the pair correlation in a self-consistent manner, they neglected the screening effct of the Coulomb interaction and the multiple Coulomb interaction between electron-hole pairs. As a result, the excitonic sharp spectral components do not reproduce in the low electron-hole densities.

The discussion was further extended to take into account the screening effect of the Coulomb interaction and the finite temperature effect by Iida et al $1 \mathrm{1}$ They considered the screening effect by the quasi-static RPA due to Haug and Schmitt-Rink analysis is based on the simple BCS-like mean field theory, so it cannot properly describe the excitonic structure in the low particle densities.

In the present article, we give a unified theory of the pump-probe spectra for highly excited semiconductors, which can be applied throughout the whole density range including the high-density electron-hole BCS state and the lowdensity excitonic BEC states. We consistently solve the combined equations of the BCS-like gap equation and the BS equation for retarded electron-hole pair Green function, which first enables us to consider the state-filling effect, the band-gap renormalization, and the weak/strong pair correlation in a unified manner. This analysis is closely related to that in Ref. 17, where the absorption-gain spectra for condensed exciton systems are calculated for various electron-hole densities and temperatures by properly taking into account the ladder diagram for vertex function. In the present article, we calculate the pump-probe spectra for various excitation conditions and systematically analyze the dependence of the spectra on the pump-light intensity and on the pump-light frequency.

As a result, we find that the electron-hole BCS state is noticeably enhanced by the intense pump-light; this result strongly suggests that the macroscopic quantum state can be observed under the strong photoexcitations. Moreover, in the high-density state generated by the small off-resonant excitation, the calculated spectrum shows a pair of sharp peaked structures at the edge of the broad absorption and stimulated emission bands. In contrast to this, we find a series of sharp exciton lines in the low density regime generated by the large off-resonant excitation. In the intermediate density generated by the strong photoexcitation, the calculated spectra considerably deviate from results obtained by the BCS-like mean-field analysis and those by the simple BS equation without pair correlation. In particular, we find the sharp spectral components originating from the excitation of the collective phase fluctuation mode of electron-hole BCS state (similar to the Anderson mode in superconductivity), which change to the exciton series with decreasing carrier density.

The article is organized as follows. In Sec. II, we derive the BS equation for the retarded Green function of electronhole pairs within the quasi-statically screened ladder approximation. In order to incorporate the pairing effect to the pump-probe spectra, we express the electron-hole pair Green function with respect to the field operators of Bogolons, that are the elementary excitation of electron-hole BCS state. We numerically calculate the pump-probe spectra by solving the BS equation in Sec. III. Finally, discussions and conclusion are given in Sec. IV.

\section{FORMULATION}

\section{A. Model Hamiltonian}

We consider an electron-hole system in a direct-gap semiconductor, which consists of the isotropic, nondegenerate parabolic conduction and valence bands. The repulsive interactions between electrons and between holes as well as the electron-hole attractive interaction are taken into account. The spin degrees of freedom are neglected to avoid the unnecessary complication due to the biexciton state. We consider that the system is in a stationary state generated by an intense monochromatic pump light with frequency $\omega_{\mathrm{L}}$; the pump light is treated as a classical field while the probe light is quantized. The model Hamiltonian is expressed as

$$
H_{\text {tot }}=H_{\mathrm{r}}+H_{\mathrm{eh}}+H_{\text {pump }}+H_{\text {probe }},
$$

where $H_{\mathrm{r}}, H_{\mathrm{eh}}, H_{\text {pump }}$ and $H_{\text {probe }}$ are the Hamiltonian of the probe photon, the electron-hole Hamiltonian, the interaction Hamiltonian between particles and pump light, and the interaction Hamiltonian between particles and 
probe light, respectively. These Hamiltonians are expressed in terms of the field operators of electrons $\left(c_{\boldsymbol{p}}\right)$, holes $\left(d_{\boldsymbol{p}}\right)$ and photons $(b)$ as follows,

$$
\begin{aligned}
H_{\mathrm{r}}= & \omega b^{\dagger} b, \\
H_{\mathrm{eh}}= & \sum_{\boldsymbol{k}}\left\{\varepsilon_{\boldsymbol{k}}^{e} c_{\boldsymbol{k}}^{\dagger} c_{\boldsymbol{k}}+\varepsilon_{\boldsymbol{k}}^{h} d_{-\boldsymbol{k}}^{\dagger} d_{-\boldsymbol{k}}\right\} \\
& +\frac{1}{2} \sum_{\boldsymbol{k}, \boldsymbol{p}, \boldsymbol{q}} V_{\boldsymbol{q}}\left\{c_{\boldsymbol{k}+\boldsymbol{q}}^{\dagger} c_{\boldsymbol{p}-\boldsymbol{q}}^{\dagger} c_{\boldsymbol{p}} c_{\boldsymbol{k}}+d_{\boldsymbol{k}+\boldsymbol{q}}^{\dagger} d_{\boldsymbol{p}-\boldsymbol{q}}^{\dagger} d_{\boldsymbol{p}} d_{\boldsymbol{k}}-2 c_{\boldsymbol{k}+\boldsymbol{q}}^{\dagger} c_{\boldsymbol{k}} d_{\boldsymbol{p}-\boldsymbol{q}}^{\dagger} d_{\boldsymbol{p}}\right\}, \\
H_{\text {pump }}= & \lambda \sum_{\boldsymbol{k}}\left\{c_{\boldsymbol{k}} d_{-\boldsymbol{k}} \exp \left(i \omega_{\mathrm{L}} t\right)+d_{-\boldsymbol{k}}^{\dagger} c_{\boldsymbol{k}}^{\dagger} \exp \left(-i \omega_{\mathrm{L}} t\right)\right\}, \\
H_{\text {probe }}= & \sum_{\boldsymbol{k}}\left\{g_{\boldsymbol{k}} b^{\dagger} c_{\boldsymbol{k}} d_{-\boldsymbol{k}}+g_{\boldsymbol{k}}^{*} d_{-\boldsymbol{k}}^{\dagger} c_{\boldsymbol{k}}^{\dagger} b\right\},
\end{aligned}
$$

where $\omega$ is the frequency of probe photon. Here $g_{\boldsymbol{k}}$ is the coupling constant between particles and the probe light; $\lambda$ is the interaction energy between particles and the pump light. The single-particle energies of electrons and holes are given by $\varepsilon_{\boldsymbol{k}}^{e}=k^{2} /\left(2 m_{e}\right)+E_{g}$, and $\varepsilon_{\boldsymbol{k}}^{h}=k^{2} /\left(2 m_{h}\right)$, respectively, where $m_{e}\left(m_{h}\right)$ and $E_{g}$ are the electron (hole) effective mass and band-gap energy, respectively. The Coulomb interaction is written as $V_{\boldsymbol{q}}=4 \pi e^{2} /\left(\epsilon_{0} q^{2}\right)$, where $\epsilon_{0}$ is the background dielectric constant of the unexcited crystal.

When we analyze a matter which is driven by a coherent light, it is convenient to convert the matter Hamiltonian to the time independent form in the so-called rotating frame. For this purpose, we use the unitary transformation given by the unitary operator 18 ,

$$
T(t)=\exp \left[-\frac{i \omega_{\mathrm{L}} t}{2} \sum_{\boldsymbol{k}}\left(c_{\boldsymbol{k}}^{\dagger} c_{\boldsymbol{k}}+d_{-\boldsymbol{k}}^{\dagger} d_{-\boldsymbol{k}}\right)\right] .
$$

The transformed Hamiltonian is written as follows,

$$
\begin{aligned}
\tilde{H}_{\mathrm{eh}}= & \sum_{\boldsymbol{k}}\left\{\xi_{\boldsymbol{k}}^{e} c_{\boldsymbol{k}}^{\dagger} c_{\boldsymbol{k}}+\xi_{\boldsymbol{k}}^{h} d_{-\boldsymbol{k}}^{\dagger} d_{-\boldsymbol{k}}\right\} \\
& +\frac{1}{2} \sum_{\boldsymbol{k}, \boldsymbol{p}, \boldsymbol{q}} V_{\boldsymbol{q}}\left\{c_{\boldsymbol{k}+\boldsymbol{q}}^{\dagger} c_{\boldsymbol{p}-\boldsymbol{q}}^{\dagger} c_{\boldsymbol{p}} c_{\boldsymbol{k}}+d_{\boldsymbol{k}+\boldsymbol{q}}^{\dagger} d_{\boldsymbol{p}-\boldsymbol{q}}^{\dagger} d_{\boldsymbol{p}} d_{\boldsymbol{k}}-2 c_{\boldsymbol{k}+\boldsymbol{q}}^{\dagger} c_{\boldsymbol{k}} d_{\boldsymbol{p}-\boldsymbol{q}}^{\dagger} d_{\boldsymbol{p}}\right\}, \\
\tilde{H}_{\text {pump }}= & \lambda \sum_{\boldsymbol{k}}\left\{c_{\boldsymbol{k}} d_{-\boldsymbol{k}}+d_{-\boldsymbol{k}}^{\dagger} c_{\boldsymbol{k}}^{\dagger}\right\}, \\
\tilde{H}_{\text {probe }}= & \sum_{\boldsymbol{k}}\left\{g_{\boldsymbol{k}} b^{\dagger} c_{\boldsymbol{k}} d_{-\boldsymbol{k}} \exp \left(-i \omega_{\mathrm{L}} t\right)+g_{\boldsymbol{k}}^{*} d_{-\boldsymbol{k}}^{\dagger} c_{\boldsymbol{k}}^{\dagger} b \exp \left(i \omega_{\mathrm{L}} t\right)\right\},
\end{aligned}
$$

where $\xi_{\boldsymbol{k}}^{e, h}=\varepsilon_{\boldsymbol{k}}^{e, h}-\omega_{\mathrm{L}} / 2$. The resulting stationary state of driven electron-hole system is controlled by the frequency of pump light $\omega_{\mathrm{L}}$, and the interaction energy $\lambda$ between pump light and carriers. The Hamiltonian $\tilde{H}_{\text {eh }}$ is formally regarded as a grand-canonical Hamiltonian of the electron-hole system, in which the chemical potential of electron-hole pairs is given by $\mu=\omega_{L}-E_{g}$.

\section{B. Optical response of probe light}

In order to evaluate the optical spectra, let us consider the equation-of-motion for expectation value of the number operator for a probe photon, $N(t)=b^{\dagger}(t) b(t)$. A perturbative calculation with respect to $\tilde{H}_{\text {probe }}$ gives

$$
\frac{d}{d t}\langle N(t)\rangle=\Pi^{<}(\omega)\langle N(t)+1\rangle-\Pi^{>}(\omega)\langle N(t)\rangle
$$

where $\langle\cdots\rangle$ indicates the expectation value with respect to the ground state of $H_{\text {tot }}$. The quantities $\Pi^{<}(\omega)$ and $\Pi^{>}(\omega)$ are the emission and the absorption rate of probe photon, respectively. The pump-probe spectrum is then given by $\Pi^{>}(\omega)-\Pi^{<}(\omega)$. The quantities $\Pi^{<}(\omega)$ and $\Pi^{>}(\omega)$ are expressed in terms of electron and hole operators as following, 


$$
\begin{aligned}
\Pi^{>}(\omega) & =\sum_{\boldsymbol{k}, \boldsymbol{p}} g_{\boldsymbol{k}}^{*} g_{\boldsymbol{p}} \int_{0}^{\infty} d t\left\langle d_{-\boldsymbol{p}}(t) c_{\boldsymbol{p}}(t) c_{\boldsymbol{k}}^{\dagger}(0) d_{-\boldsymbol{k}}^{\dagger}(0)\right\rangle_{0} \mathrm{e}^{i\left(\omega-\omega_{\mathrm{L}}\right) t}+c . c . \\
& =-2 \operatorname{Im} G^{>}\left(\omega-\omega_{\mathrm{L}}+i \gamma\right), \\
\Pi^{<}(\omega) & =\sum_{\boldsymbol{k}, \boldsymbol{p}} g_{\boldsymbol{k}}^{*} g_{\boldsymbol{p}} \int_{0}^{\infty} d t\left\langle c_{\boldsymbol{k}}^{\dagger}(0) d_{-\boldsymbol{k}}^{\dagger}(0) d_{-\boldsymbol{p}}(t) c_{\boldsymbol{p}}(t)\right\rangle_{0} \mathrm{e}^{i\left(\omega-\omega_{\mathrm{L}}\right) t}+c . c . \\
& =-2 \operatorname{Im} G^{<}\left(\omega-\omega_{\mathrm{L}}+i \gamma\right),
\end{aligned}
$$

where $\gamma$ is the exciton decay constant and $\langle\cdots\rangle_{0}$ stands for the expectation value with respect to the ground state of $H=\tilde{H}_{\text {eh }}+\tilde{H}_{\text {pump }}$. Here $G^{>}(\omega)$ and $G^{<}(\omega)$ are the Fourier transform of the correlation functions defined by

$$
\begin{aligned}
& G^{>}(t)=-i \Theta(t) \sum_{\boldsymbol{k}, \boldsymbol{p}} g_{\boldsymbol{k}}^{*} g_{\boldsymbol{p}}\left\langle d_{-\boldsymbol{p}}(t) c_{\boldsymbol{p}}(t) c_{\boldsymbol{k}}^{\dagger}(0) d_{-\boldsymbol{k}}^{\dagger}(0)\right\rangle_{0}, \\
& G^{<}(t)=-i \Theta(t) \sum_{\boldsymbol{k}, \boldsymbol{p}} g_{\boldsymbol{k}}^{*} g_{\boldsymbol{p}}\left\langle c_{\boldsymbol{k}}^{\dagger}(0) d_{-\boldsymbol{k}}^{\dagger}(0) d_{-\boldsymbol{p}}(t) c_{\boldsymbol{p}}(t)\right\rangle_{0} .
\end{aligned}
$$

The pump-probe spectrum is given by

$$
A(\omega)=-2 \operatorname{Im} G_{\mathrm{R}}\left(\omega-\omega_{\mathrm{L}}+i \gamma\right),
$$

where $G_{\mathrm{R}}(\omega)$ is the Fourier transform of the retarded Green function for electron-hole pairs given by

$$
G_{\mathrm{R}}(t) \equiv G^{>}(t)-G^{<}(t) .
$$

In the following analysis, we calculate $G_{\mathrm{R}}(t)$ by solving the BS equation.

\section{BCS-like gap equation for electron-hole systems}

In order to incorporate the electron-hole pair correlation, let us introduce the Bogoliubov transformation given by,

$$
\begin{aligned}
c_{k} & =u_{\boldsymbol{k}} \alpha_{k}+v_{\boldsymbol{k}} \beta_{-\boldsymbol{k}}^{\dagger}, \\
d_{-\boldsymbol{k}} & =u_{\boldsymbol{k}} \beta_{-\boldsymbol{k}}-v_{\boldsymbol{k}} \alpha_{\boldsymbol{k}}^{\dagger},
\end{aligned}
$$

where $\alpha_{\boldsymbol{k}}$ and $\beta_{-\boldsymbol{k}}$ are the annihilation operators for new quasiparticles (Bogolons). The Bogoliubov parameters $u_{\boldsymbol{k}}$ and $v_{\boldsymbol{k}}$ are subject to the condition, $u_{\boldsymbol{k}}^{2}+v_{\boldsymbol{k}}^{2}=1$.

In the following analysis, it is convenient to introduce the two-component operator $\phi_{\boldsymbol{k}}=\left(\alpha_{\boldsymbol{k}}, \beta_{-\boldsymbol{k}}^{\dagger}\right)$ and the $2 \times 2$ unit matrix $\boldsymbol{\tau}_{0}$ and Pauli matrices $\boldsymbol{\tau}_{j}(j=1,2,3)$. The Hamiltonian $H=\tilde{H}_{\text {eh }}+\tilde{H}_{\text {pump }}$ is expressed in terms of $\phi_{\boldsymbol{k}}$ as follows,

$$
H=\sum_{\boldsymbol{k}} \mathcal{E}_{\boldsymbol{k}}^{\mu}\left(\phi_{\boldsymbol{k}}^{\dagger} \boldsymbol{\tau}_{\mu} \phi_{\boldsymbol{k}}\right)+\frac{1}{2} \sum_{\boldsymbol{k}, \boldsymbol{p}, \boldsymbol{q}} W_{\boldsymbol{k}, \boldsymbol{p}}^{\mu, \nu}(\boldsymbol{q})\left(\phi_{\boldsymbol{k}+\boldsymbol{q}}^{\dagger} \boldsymbol{\tau}_{\mu} \phi_{\boldsymbol{k}}\right)\left(\phi_{\boldsymbol{p}-\boldsymbol{q}}^{\dagger} \boldsymbol{\tau}_{\nu} \phi_{\boldsymbol{p}}\right)
$$

where the summation convention with respect to the indices $\mu, \nu=0,1, \cdots, 3$ is employed. Introducing the coherence factors, $C_{\boldsymbol{k}, \boldsymbol{p}}^{(\mu)}(\mu=0,1, \cdots, 3)$, given by

$$
\begin{aligned}
& C_{\boldsymbol{k}, \boldsymbol{p}}^{(0)}=u_{\boldsymbol{k}} u_{\boldsymbol{p}}+v_{\boldsymbol{k}} v_{\boldsymbol{p}}, \\
& C_{\boldsymbol{k}, \boldsymbol{p}}^{(1)}=u_{\boldsymbol{k}} v_{\boldsymbol{p}}+v_{\boldsymbol{k}} u_{\boldsymbol{p}}, \\
& C_{\boldsymbol{k}, \boldsymbol{p}}^{(2)}=u_{\boldsymbol{k}} u_{\boldsymbol{p}}-v_{\boldsymbol{k}} v_{\boldsymbol{p}}, \\
& C_{\boldsymbol{k}, \boldsymbol{p}}^{(3)}=u_{\boldsymbol{k}} v_{\boldsymbol{p}}-v_{\boldsymbol{k}} u_{\boldsymbol{p}},
\end{aligned}
$$

and $\mathcal{E}_{\boldsymbol{k}}^{\mu}$ can be expressed as follows, 


$$
\begin{aligned}
\mathcal{E}_{\boldsymbol{k}}^{0} & =\frac{1}{2}\left(\xi_{\boldsymbol{k}}^{(e)}-\xi_{\boldsymbol{k}}^{(h)}\right), \\
\mathcal{E}_{\boldsymbol{k}}^{1} & =\lambda C_{\boldsymbol{k}, \boldsymbol{k}}^{(2)}+\frac{1}{2}\left(\xi_{\boldsymbol{k}}^{(e)}+\xi_{\boldsymbol{k}}^{(h)}-\sum_{\boldsymbol{q}} V_{\boldsymbol{q}}\right) C_{\boldsymbol{k}, \boldsymbol{k}}^{(1)} \\
\mathcal{E}_{\boldsymbol{k}}^{2} & =0 \\
\mathcal{E}_{\boldsymbol{k}}^{3} & =\frac{1}{2}\left(\xi_{\boldsymbol{k}}^{(e)}+\xi_{\boldsymbol{k}}^{(h)}-\sum_{\boldsymbol{q}} V_{\boldsymbol{q}}\right) C_{\boldsymbol{k}, \boldsymbol{k}}^{(2)}-\lambda C_{\boldsymbol{k}, \boldsymbol{k}}^{(1)}
\end{aligned}
$$

The quantity $W_{\boldsymbol{k}, \boldsymbol{p}}^{\mu, \nu}(\boldsymbol{q})$ is written as follows,

$$
W_{\boldsymbol{k}, \boldsymbol{p}}^{\mu, \nu}(\boldsymbol{q})=V_{\boldsymbol{q}}\left(\begin{array}{ccccc}
C_{\boldsymbol{k}+\boldsymbol{q}, \boldsymbol{k}}^{(0)} C_{\boldsymbol{p}-\boldsymbol{q}, \boldsymbol{p}}^{(0)} & 0 & i C_{\boldsymbol{k}+\boldsymbol{q}, \boldsymbol{k}}^{(0)} C_{\boldsymbol{p}-\boldsymbol{q}, \boldsymbol{p}}^{(3)} & 0 \\
0 & 0 & 0 & 0 \\
i C_{\boldsymbol{k}+\boldsymbol{q}, \boldsymbol{k}}^{(3)} C_{\boldsymbol{p}-\boldsymbol{q}, \boldsymbol{p}}^{(0)} & 0 & -C_{\boldsymbol{k}+\boldsymbol{q}, \boldsymbol{k}}^{(3)} C_{\boldsymbol{p}-\boldsymbol{q}, \boldsymbol{p}}^{(3)} & 0 \\
0 & 0 & 0 & 0
\end{array}\right)_{\mu, \nu} .
$$

The Bogoliubov parameters are determined by the variational method. We minimize the expectation value of the Hamiltonian, $\langle H\rangle_{0}$, under the charge neutrality condition, $\sum_{\boldsymbol{k}}\left\{\left\langle c_{\boldsymbol{k}}^{\dagger} c_{\boldsymbol{k}}\right\rangle_{0}+\left\langle d_{-\boldsymbol{k}}^{\dagger} d_{-\boldsymbol{k}}\right\rangle_{0}\right\}=0$, which gives

$$
u_{\boldsymbol{k}}^{2}=\frac{1}{2}\left(1+\frac{\zeta_{\boldsymbol{k}}}{E_{\boldsymbol{k}}}\right), v_{\boldsymbol{k}}^{2}=\frac{1}{2}\left(1-\frac{\zeta_{\boldsymbol{k}}}{E_{\boldsymbol{k}}}\right) .
$$

Since $\left\langle c_{\boldsymbol{k}}^{\dagger} c_{\boldsymbol{k}}\right\rangle_{0}=\left\langle d_{-\boldsymbol{k}}^{\dagger} d_{-\boldsymbol{k}}\right\rangle_{0}=v_{\boldsymbol{k}}^{2}$, the physical meaning of $v_{\boldsymbol{k}}^{2}$ is the distribution function of electrons and holes. In the above equation, $\zeta_{\boldsymbol{k}}, \Delta_{\boldsymbol{k}}$, and $E_{\boldsymbol{k}} \equiv \sqrt{\zeta_{\boldsymbol{k}}^{2}+\Delta_{\boldsymbol{k}}^{2}}$ are the renormalized energy of electron-hole pair, the BCS-like energy gap, and the single particle excitation energy of Bogolon, respectively. The quantities $\zeta_{\boldsymbol{k}}$ and $\Delta_{\boldsymbol{k}}$ satisfy the following self-consistent equations

$$
\begin{aligned}
\zeta_{\boldsymbol{k}} & =\left(\frac{k^{2}}{2 m^{*}}-\omega_{\mathrm{L}}+E_{g}\right)-2 \sum_{\boldsymbol{p}} V_{\boldsymbol{k}-\boldsymbol{p}} v_{\boldsymbol{p}}^{2} \\
& =\left(\frac{k^{2}}{2 m^{*}}-\omega_{\mathrm{L}}+E_{g}\right)-\sum_{\boldsymbol{p}} V_{\boldsymbol{k}-\boldsymbol{p}}\left(1-\frac{\zeta_{\boldsymbol{p}}}{E_{\boldsymbol{p}}}\right) \\
\Delta_{\boldsymbol{k}} & =2 \lambda-4 \sum_{\boldsymbol{p}} V_{\boldsymbol{k}-\boldsymbol{p}} u_{\boldsymbol{p}} v_{\boldsymbol{p}} \\
& =2 \lambda-2 \sum_{\boldsymbol{p}} V_{\boldsymbol{k}-\boldsymbol{p}} \frac{\Delta_{\boldsymbol{p}}}{E_{\boldsymbol{p}}}
\end{aligned}
$$

where $m^{*}=m_{e} m_{h} /\left(m_{e}+m_{h}\right)$ is the reduced mass of electron-hole pairs. The second term of the right-hand side of Eq. (15a) expresses the band renormalization effect arising from the electron (hole) exchange interaction. The Eq. (15b) is the BCS-like gap equation where the BCS-like energy gap formation induced by the pump-light is taken into account.

In order to obtain the physical insight into the BCS-like gap equation, we express the BCS_like gap equation, Eq. (15), in terms of the electron-hole pair wave function with zero center-of-mass momentum $\mathbb{L}^{1}, \psi_{\boldsymbol{k}}=\left\langle c_{\boldsymbol{k}} d_{-\boldsymbol{k}}\right\rangle=$ $\Delta_{\boldsymbol{k}} /\left(2 E_{\boldsymbol{k}}\right)$. Making use of Eq. (14), the BCS-like gap equation can be rewritten as

$$
\left\{\frac{k^{2}}{2 m^{*}}-\left(\omega_{\mathrm{L}}-E_{g}\right)-2 \sum_{\boldsymbol{p}} V_{\boldsymbol{k}-\boldsymbol{p}} v_{\boldsymbol{p}}^{2}\right\} \psi_{\boldsymbol{k}}-\left(1-2 v_{\boldsymbol{k}}^{2}\right) \sum_{\boldsymbol{p}} V_{\boldsymbol{k}-\boldsymbol{p}} \psi_{\boldsymbol{p}}=\left(1-2 v_{\boldsymbol{k}}^{2}\right) \lambda .
$$

This equation is reduced to the Wannier equation in the limit of low electron-hole densities and $\lambda \rightarrow 0$, because $v_{\boldsymbol{k}}^{2}$ is the pair distribution function. Therefore the BCS-like pair theory is able to properly describe the relative wave-function of excitons in the low density case as well as the BCS-like pair states in the high-density case.

\section{The Bethe-Salpeter equation for electron-hole pair Green function}

As described above, the optical spectrum is obtained by the evaluation of the electron-hole retarded Green function $G_{\mathrm{R}}(t)$, which can be rewritten in terms of Bogoliubov parameters as, 


$$
G_{\mathrm{R}}(t)=\sum_{\boldsymbol{k}, \boldsymbol{p}} g_{\boldsymbol{k}}^{*} g_{\boldsymbol{p}} \boldsymbol{K}_{\boldsymbol{k}} \mathcal{G}_{\boldsymbol{k}, \boldsymbol{p}}(t) \boldsymbol{K}_{\boldsymbol{p}}
$$

where $\boldsymbol{K}_{\boldsymbol{k}} \equiv\left(u_{\boldsymbol{k}}^{2}-v_{\boldsymbol{k}}^{2}, i\right)$ is the two-component vector. $\mathcal{G}_{\boldsymbol{k}, \boldsymbol{p}}(t)$ is the $2 \times 2$ matrix retarded Green function whose $(\mu, \nu)$ component is given by

$$
\left(\mathcal{G}_{\boldsymbol{k}, \boldsymbol{p}}(t)\right)_{\mu, \nu}=-i \Theta(t)\left\langle\left[\left(\phi_{\boldsymbol{k}}^{\dagger}(t) \boldsymbol{\tau}_{\mu} \phi_{\boldsymbol{k}}(t)\right),\left(\phi_{\boldsymbol{p}}^{\dagger}(0) \boldsymbol{\tau}_{\nu} \phi_{\boldsymbol{p}}(0)\right)\right]\right\rangle
$$

where $\mu, \nu=1,2$. The function $\mathcal{G}_{\boldsymbol{k}, \boldsymbol{p}}(\omega)$ satisfies the following BS equation,

$$
\left(\omega \boldsymbol{\tau}_{0}+E_{\boldsymbol{k}} \boldsymbol{\tau}_{2}\right) \mathcal{G}_{\boldsymbol{k}, \boldsymbol{p}}(\omega)-\sum_{\boldsymbol{k}^{\prime}} V_{\boldsymbol{k}-\boldsymbol{k}^{\prime}}\left(C_{\boldsymbol{k}, \boldsymbol{k}^{\prime}}^{(0) 2} \boldsymbol{\tau}_{2}+i C_{\boldsymbol{k}, \boldsymbol{k}^{\prime}}^{(3) 2} \boldsymbol{\tau}_{1}\right) \mathcal{G}_{\boldsymbol{k}^{\prime}, \boldsymbol{p}}(\omega)=\boldsymbol{\tau}_{2} \delta_{\boldsymbol{k}, \boldsymbol{p}}
$$

This BS equation is obtained by linearizing the equation-of-motion for $\mathcal{G}_{\boldsymbol{k}, \boldsymbol{p}}(t)$. The second term on the left-hand side of Eq. (19) describes the effect of multiple Coulomb interaction between Bogolons, which is attractive in the low electron-hole density limit.

The vertex part proportional to $C_{\boldsymbol{k}, \boldsymbol{p}}^{(0) 2}$ reflects the state-filling effect and the part proportional to $C_{\boldsymbol{k}, \boldsymbol{p}}^{(3) 2}$ arises because of the electron-hole pair correlation. It should be emphasized that this term describes the deviation from the BCS-like mean-field theory, and give rise to the sharp excitonic structures in the pump-probe spectrum.

\section{E. Screening effect}

We consider the screening effect within the quasi-static RPA following the work by Haug and Schmitt-Rink 1 . The BCS-like gap equation, Eq. (15), is rewritten as follows,

$$
\begin{gathered}
\zeta_{\boldsymbol{k}}=\left(\frac{k^{2}}{2 m^{*}}-\omega_{\mathrm{L}}+E_{g}\right)-2 \sum_{\boldsymbol{p}} V_{\boldsymbol{k}-\boldsymbol{p}}^{\mathrm{s}} v_{\boldsymbol{p}}^{2}+\sum_{\boldsymbol{q}}\left\{V_{\boldsymbol{q}}^{\mathrm{s}}-V_{\boldsymbol{q}}\right\}, \\
\Delta_{\boldsymbol{k}}=2 \lambda-4 \sum_{\boldsymbol{p}} V_{\boldsymbol{k}-\boldsymbol{p}}^{\mathrm{s}} u_{\boldsymbol{p}} v_{\boldsymbol{p}}
\end{gathered}
$$

where $V_{\boldsymbol{q}}^{\mathrm{s}}$ is the quasi-statically screened Coulomb potential. Here the second term on the right-hand side of Eq. (20a) represent the screened exchange effect and the third term being the Coulomb-hole effect. The BS equation, Eq. (19), is rewritten as

$$
\left(\omega \boldsymbol{\tau}_{0}+E_{\boldsymbol{k}} \boldsymbol{\tau}_{2}\right) \mathcal{G}_{\boldsymbol{k}, \boldsymbol{p}}(\omega)-\sum_{\boldsymbol{k}^{\prime}} V_{\boldsymbol{k}-\boldsymbol{k}^{\prime}}^{\mathrm{s}}\left(C_{\boldsymbol{k}, \boldsymbol{k}^{\prime}}^{(0) 2} \boldsymbol{\tau}_{2}+i C_{\boldsymbol{k}, \boldsymbol{k}^{\prime}}^{(3) 2} \boldsymbol{\tau}_{1}\right) \mathcal{G}_{\boldsymbol{k}^{\prime}, \boldsymbol{p}}(\omega)=\boldsymbol{\tau}_{2} \delta_{\boldsymbol{k}, \boldsymbol{p}}
$$

We employ the tractable expression for the dielectric function given by the single-plasmon-pole approximation, which is known to produce the relatively good self-energy corrections 1612 The explicit expression for the screened potential is given by,

$$
V_{\boldsymbol{q}}^{\mathrm{s}}=\frac{4 \pi e^{2}}{\epsilon_{\boldsymbol{q}} q^{2}}, \epsilon_{\boldsymbol{q}}^{-1}=\epsilon_{0}^{-1}\left(1-\frac{\omega_{\mathrm{pl}}^{2}}{\omega_{\boldsymbol{q}}^{2}}\right),
$$

where $\omega_{\mathrm{pl}}=4 \pi n e^{2} /\left(\epsilon_{0} m^{*}\right)$ is the plasma frequency, and $n$ is the electron-hole pair density. The dispersion of the effective plasmon mode is given by 20

$$
\omega_{\boldsymbol{q}}^{2}=\omega_{\mathrm{pl}}^{2}\left(1+\frac{q^{2}}{k_{\mathrm{TF}}^{2}}\right)+\left(\frac{q^{2}}{4 m^{*}}\right)^{2}+G_{\mathrm{eff}}^{2},
$$

where $k_{\mathrm{TF}}=\left\{16 m^{*} e^{2} /\left(\pi \epsilon_{0}\right)\right\}\left(6 \pi^{2} n\right)^{-1 / 3}$ is the Thomas-Fermi wave number, and the effective gap $G_{\text {eff }}$ is set to the minimum energy of the Bogolon pair excitation. 


\section{NUMERICAL ANALYSIS}

\section{A. Solution of the BCS-like gap equation and the resonatorless optical bistability}

In the following analysis, we use the units where the exciton binding energy and the exciton Bohr radius being unity. For simplicity, we assume that the electron and hole masses are identical, i.e., $m_{e}=m_{h}$.

As a first step in our numerical calculations, we iteratively solve the BCS-like gap equation, Eq. (20). Here we note that the quasi-stationary state of this optically driven electron-hole system is controlled by changing $\omega_{\mathrm{L}}$ and $\lambda$.

We first show the calculated mean interparticle distance, $r_{\mathrm{s}}=(4 \pi n / 3)^{-1 / 3}$, as a function of pump-frequency in Fig. 1, where $n$ is the electron-hole density. We find the resonatorless optical bistability in which the two stable states exist for the same pump-frequency, as shown in Ref. 16. With increasing the interaction energy $\lambda$ between carriers and pump-light, the bistability region of $\omega_{\mathrm{L}}$ becomes narrow and, the bistable behavior disappears for $\lambda \gtrsim 0.3$.

For convenience in the following discussion, we show in Fig. 2 the pump-frequency $\omega_{\mathrm{L}}$ dependence of the effectivegap $G_{\text {eff }}$, which is defined by the minimum value of the single-particle excitation energy $E_{\boldsymbol{k}}$. In the high electron-hole density generated by the resonant excitations $\left(\omega_{\mathrm{L}}-E_{g}>0\right)$, the weak electron-hole pair correlation predominates, in which the BCS-like energy gap is formed at the quasi-Fermi level. With decreasing the pump-frequency, the BCS-like gap $\Delta_{k}$ continues to exist even for the off-resonance cases especially in the higher density side of the bistable region. This is because the system undergoes the resonance condition because of the band-gap renormalization effect. We should remind that the BCS-like gap for degenerate electron-hole systems is much smaller than the exciton binding energy. The special attention should be paid to the fact that the magnitude of the BCS-like gap becomes very large with increasing $\lambda$, which is shown in Fig. 22. This behavior arises because the electron-hole pair correlation is strongly enhanced by the coherent pump-light, which was pointed out in Ref. 15 as the "light enhanced electronhole correlation". On the contrary, in the low electron-hole density generated by the large off-resonant excitations $\left(\omega_{\mathrm{L}}-E_{g} \ll 0\right)$, the contribution of the Coulomb interaction to $G_{\text {eff }}$ is disregarded, and $G_{\text {eff }}$ is approximated to $E_{g}-\omega_{\mathrm{L}}$.

\section{B. Pump-probe spectra under weak photoexcitations}

Now, let us consider the pump-probe spectrum $A(\omega)$ given by Eq. (7). The positive and negative values of $A(\omega)$ imply the absorption and the stimulated emission of the probe light, respectively. When we numerically solve the BS equation, Eq. (21), the usual singularity-removal method 14 is inappropriate because the singularity of the retarded Green function considerably depends on the electron-hole density. This fact forces us to deal with a giant matrix (about $3000 \times 3000$ in dimension) to obtain good overall accuracy. In the present analysis we numerically evaluate the eigenvalues and eigenvectors of the stability matrix 21 of the BS kernel in Eq. (21).

In Figs. 3 5, we show the calculated spectra for several values of $\omega_{\mathrm{L}}$ and $\lambda=0.001$, in which the bistability region exists. Figure 3 is the spectrum for a higher density state $\left(\omega_{\mathrm{L}}-E_{g}=-0.5, r_{\mathrm{s}}=0.87\right)$ outside the bistability region, and Fig. 1 is that for a lower density state $\left(\omega_{\mathrm{L}}-E_{g}=-4.0, r_{\mathrm{s}}=140\right)$ outside the bistability region. The spectra inside the bistability region $\left(\omega_{\mathrm{L}}-E_{g}=-2.5\right)$ in higher $\left(r_{\mathrm{s}}=1.0\right)$ and lower $\left(r_{\mathrm{s}}=86\right)$ density states are shown in Fig. 5.

In the high-density state (Fig. 3) outside the bistability region, the strong stimulated emission, and the large red-shift of the band-edge due tp the band renormalization effect is found. The transparent region arising from the BCS-like energy gap formation 16 is almost vanishing in this choice of parameters, because the BCS-like gap is extremely small as shown in Fig. 2. However, this does not imply that the BCS-like gap is not formed; actually, around $\omega \simeq \omega_{\mathrm{L}}$, we find a pair of sharp peaked structures in the pump-probe spectrum, which originates from the singular behavior of the density-of-states accompanied by the BCS-like energy gap formation due to the many-body effect. It should be noted that this anomaly is weak in the BCS-like mean-field calculation as shown by the dashed line in Fig. 3, and the BS equation, Eq. (21), properly describes the strong electron-hole pair correlation.

In contrast to Fig. 3, the pump-probe spectrum in the low-density state outside the bistability region exhibits the qualitatively different profile. We find, in Fig. 1, a series of sharp absorption lines corresponding to the $1 \mathrm{~S}, 2 \mathrm{~S}$... exciton states, and the calculated spectrum is reduced to the one given by the Elliott formulal in the low density limit. However, it should be noted that the present analysis is based on the electron-hole many-body theory including the strong electron-hole pair correlation, and the large deviations from the square-root dependence of $A(\omega)$ is observed close to the band-edge. In addition, it should be stressed that the BCS-like decoupling approximation for electronhole pair Green function cannot reproduce the excitonic structure, even though the BCS-like gap equation properly describes the internal motion of excitons both in the high and low density limits. 
Inside the bistability region, the high- and the low-density behavior appears for the same pump frequency $\omega_{\mathrm{L}}$, as shown in Fig. 5. We should recall that this bistability arises from the instability due to the positive feedback between the optical pumping and the band-gap renormalization.

\section{The dependence of the pump-probe spectra on the pump-light intensity}

In the following, let us discuss the dependence of the pump-probe spectrum on the interaction energy between carriers and pump-light $\lambda$. We show in Fig. 6 the calculated spectra for three states: $\lambda=0.1,0.2$ and 0.3 . Here the pump-light frequency is chosen as $\omega_{\mathrm{L}}-E_{g}=-0.5$.

As in the case of Fig. 3, we find the stimulated emission in $\omega<\omega_{\mathrm{L}}$, and the absorption components in $\omega>\omega_{\mathrm{L}}$. As shown in Fig. 1, the mean interparticle distance is $r_{\mathrm{s}} \approx 0.87$, irrespective of the magnitude of $\lambda$, because the electron-hole recombination rate is assumed to be much smaller than the Rabi frequency. Between the absorption and the emission parts, we find the transparent region originating from the BCS-like gap formation, which is extremely enhanced by the pump-light excitation. Conversely, the Rabi splitting of the dressed semiconductor states by the strong pump light is combined with the electron-hole Coulomb interaction to form extremely large BCS-like gap. In the large off-resonance case (and the low-density state in the bistability region), the energy gap becomes insensitive to $\lambda$, as shown in Fig. 2.

With increasing $\lambda$, the broad absorption (emission) component splits into a sharp absorption (emission) line and the broad absorption (emission) band. In the following discussion, we call these sharp stimulated emission and the absorption lines as $A_{\mathrm{e}}$-line and $A_{\mathrm{a}}$-line, respectively. The $A_{\mathrm{e}^{-}}$and $A_{\mathrm{a}}$-lines appear inside the transparent region given by the BCS-like mean field calculation which is shown by the dashed curves in Figs. 35 and Figs. 7 10. The $A_{\mathrm{e}^{-}}$and $A_{\mathrm{a}}$-lines are manifestation of the optical transition accompanied by the excitation of the collective phase fluctuation mode in the electron-hole BCS state, which corresponds to the Anderson mode in superconductivityl. The attention should be paid to the fact that this mode is induced by the strong pump-light, which is reduced to the band edge singularity for the weak pump-light as discussed above. That is to say, these lines do not simply arise from the conventional excitonic processes, because the exciton picture breaks down in this density regime and their spectral positions are not located in the semiconductor band gap but in the BCS-like gap. Since the collective

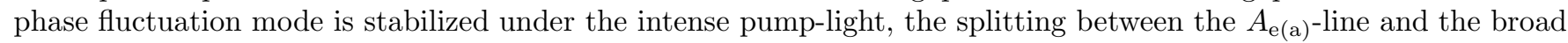
stimulated emission (absorption) component becomes larger with increasing $\lambda$.

\section{Pump-probe spectra under strong photoexcitations}

In the following, we discuss the dependence of the pump-probe spectra on the pump-light frequency $\omega_{\mathrm{L}}$ in the highdensity states under the strong photoexcitations. Figures $7-10$ depict the spectra for $\lambda=0.3$, where the interaction energy between the pump-light and carriers is sufficiently strong as to suppress the resonatorless optical bistability 16 .

We show in Fig. 7 the calculated spectrum for $\omega_{\mathrm{L}}-E_{g}=-1.8$, in which the mean interparticle distance is $r_{\mathrm{s}}=1.0$. In spite of the relatively large off-resonance, we find the $A_{\mathrm{e}}$-line $\left(A_{\mathrm{a}}\right.$-line) in the higher (lower) frequency side of the broad emission (absorption) component as shown in Fig. 6; this is because the large band renormalization preserves the system in the resonance condition.

We next show the spectra for $\omega_{\mathrm{L}}-E_{g}=-2.75$ (Fig. 8), $\omega_{\mathrm{L}}-E_{g}=-2.9$ (Fig. 9) and $\omega_{\mathrm{L}}-E_{g}=-3.2$ (Fig. 10), where the mean interparticle distances are $r_{\mathrm{s}}=1.4, r_{\mathrm{s}}=1.8$ and $r_{\mathrm{s}}=2.3$, respectively. Making a comparison between Figs. 19- we find that the broad emission (absorption) component splits into a series of sharp emission (absorption) lines and a broad emission (absorption) band with decreasing the pump-light frequency $\omega_{\mathrm{L}}$. The spectral positions of the new series of sharp emission lines and the $A_{\mathrm{e}}$-line slightly shift towards longer wavelength with decreasing the pump-light frequency, because the effective gap $G_{\text {eff }}$ increases as shown in Fig. 2. We find, in Figs. 9 - 10 that the intensities of this series of sharp emission lines and the broad emission band become weak as the pump-light frequency decreases. It is pointed out that neither this series nor the $A_{\mathrm{e} / \mathrm{a}}$-line is obtained by the BCS-like pairing theory even with the RPA correction.

We should be noted that those series of sharp emission/absorption components reflect the crossover behavior from the optically induced collective fluctuation to the exciton real-space pairing with multiple bound states (Wannier series).

In the large off-resonant excitation (Figs. 9 10), a series of sharp absorption components including the $A_{\mathrm{a}-\text { line can }}$ be regarded as the exciton lines, where the excitonic real-space pairing in the many exciton system play the major role. Moreover, the spectral positions for emission components are well described by the nonlinear optical processes in many exciton systems (the exciton transition by the combination of the two pump-photon absorption and one 
probe-photon emission) rather than the pair recombination processes accompanied by the excitation of the collective phase fluctuation mode in the electron-hole BCS state. In fact, we find in Figs. 910 that the magnitude of the effective gap $G_{\text {eff }}$ is approximately equal to $E_{g}-\omega_{\mathrm{L}}$, and the frequency $(\omega)$ of the $A_{\mathrm{e}}$-line and the series of sharp emission lines are given by $2 \omega_{\mathrm{L}}-\omega=E_{g}-E_{n, x}$, where $E_{n, x}$ with $n=1,2, \cdots$ is the binding energy of the $n \mathrm{~S}$ exciton and $n=1$ $(n=2,3, \cdots)$ corresponds to $A_{\mathrm{e}}$-line (the series of sharp emission lines).

On the contrary, Fig. 8 can be regarded as the intermediate state between the light-enhanced electron-hole BCS state and the many exciton system. This is because the broad band and the sharp lines coexist in the stimulated emission spectrum, and the $A_{\mathrm{e}}$-line has the intermediate character between the collective phase fluctuation and the above-mentioned nonlinear optical process for the 1S exciton.

We next compare the calculated spectra given by the present theory and by the BCS-like mean-field theory. Figures 89 indicate that the spectral positions of the series of sharp emission and absorption lines appear inside the transparent region given by the BCS-like mean-field calculation. It should be noted here that the origin of those series of sharp emission and absorption lines including $A_{\mathrm{e}^{-}}$and $A_{\mathrm{a}}$-lines are different from that of pair of peaked structures in the BCS-like mean-field calculation, which originate from the singularity of the density-of-states for the Bogolon excitation. In addition, we find, in Fig. 8, that the intensity of the broad stimulated emission component given by the present theory is much weaker than that of the corresponding spectral component predicted by the BCS-like mean-field calculation, and the intensity of the broad emission band is almost vanishing in Figs. 910 .

\section{CONCLUSION}

We present a many-body theory for pump-probe spectra in highly excited semiconductors which is applicable throughout the whole electron-hole density states; the high-density electron-hole BCS state and the low density excitonic BEC state are contained as the limits. The present theory is based on the BCS-like pairing theory combined with the BS equation for electron-hole pair propagator, which first enables us to consider the state-filling effect, the band-gap renormalization and the strong/weak pair correlation in a unified manner.

We calculate the pump-probe spectra under the weak photoexcitations. In the high density state generated by a small off-resonant pumping, we first show that a pair of sharp peaked structure near the pump-light frequency is considerably enhanced by the strong pair correlation. On the other hand, in the low density state given by a large off-resonant pumping, we find the excitonic absorptiqn structures near the band-edge, which is missing in the BCS-like mean-field theory even with the RPA correction 15,16 .

We next analyze the dependence of the spectra on the pump-light intensities. It is shown that the electron-hole BCS state is stabilized and the BCS-like gap is distinctly enhanced by the intense pump-light. This result strongly suggests that the macroscopic quantum phenomena, such as excitonic superfluidity, can be observed under the strong photoexcitation.

With increasing the pump-light intensity, we find that those new sharp emission and absorption lines $\left(A_{\mathrm{e}^{-}}\right.$and $A_{\mathrm{a}}$-lines) split from the broad emission and absorption components, respectively. These new lines originate from the optical transition accompanied by the collective phase fluctuation mode in the electron-hole BCS state, which corresponds to the Anderson mode in superconductivityt.

Finally, we analyze the dependence of the calculated spectra on the pump-light frequency under the strong photoexcitation, and show that the collective phase fluctuation mode continuously changes to the exciton mode as the pump-light frequency decreases.

\section{ACKNOWLEDGMENT}

This work is partially supported by a Grant-in-Aid for Scientific Research on priority areas, "Photo-induced Phase Transition and Their Dynamics" from the Ministry of Education, Science, Culture and Sports of Japan.

$\dagger$ Electronic Address: inagaki@ms.aist-nara.ac.jp

${ }^{1}$ As a reference, see for example, H. Haug and S. Schmitt-Rink, Prog. Quantum Electron. 9, 3 (1988).

${ }^{2}$ See, e.g., E. Hanamura and H. Haug, Phys. Rep. 33, 209 (1977). 
${ }^{3}$ E. Fortin, S. Farad and A. Mysyrowicz, Phys. Rev. Lett. 70, 3951 (1993); A. Mysyrowicz, E. Benson and E. Fortin, Phys. Rev. Lett. 77, 896 (1996).

${ }^{4}$ H. Kondo, T. Kawai, T. Karasawa, I. Akai and T. Komatsu, J. Luminescence 66 \& 67, 448 (1996); H. Kondo, H. Mino, I. Akai and T. Karasawa, Phys. Rev. B 58, 13835 (1998).

${ }^{5}$ L. V. Keldysh and Yu. V. Kopaev, Sov. Phys. Solid State 6, 2219 (1965); L. V. Keldysh and A. N. Kozlov, Sov. Phys. JETP 27, 521 (1968); C. Comte and P. Nozières, J. Phys. (Paris) 43, 1069 (1982); P. Nozières and C. Comte, J. Phys. (Paris) 43, $1083(1982)$.

${ }^{6}$ T. J. Inagaki, M. Aihara and A. Takahashi, preprint.

${ }^{7}$ P. W. Anderson, Phys. Rev. 112, 1900 (1958); G. Rickayzen, Phys. Rev. 115, 795 (1959); A. Bardasis and J. R. Schrieffer, Phys. Rev. 121, 1050 (1961).

${ }^{8}$ See, e.g., N. Nakanishi, Prog. Theor. Phys. Suppl. (Kyoto) 43, 1 (1969); 95, 1 (1988).

${ }^{9}$ C. Benoit à la Guillaume, J. -M. Debever and F. Salvan, Phys. Rev. 177, 567 (1969); J. Bille, H. Liebing and P. Mengel, Phys. Stat. Sol. B 53, 353 (1972).

${ }^{10}$ P. Yu, A. K. Tang, G. K. L. Wong, M. Kawasaki and Y. Segawa, in Proceedings of the 23rd International Conference on the Physics of Semiconductors, edited by M. Scheffler and R. Zimmermann (World Scientific, Singapore, 1996$)$, Vol. 2 , p. 1453.

11 Z. K, Tang et al., Appl. Phys. Lett. 72, 3270 (1998).

12 J. P. Löwenau, S. Schmitt-Rink and H. Haug, Phys. Rev. Lett. 49, 1511 (1982).

${ }^{13}$ S. Schmitt-Rink, J. P. Löwenau and H. Haug, Z. Phys. B 47, 13 (1982); S. Schmitt-Rink, C. Ell and H. Haug, Phys. Rev. B 33, 1183 (1986).

${ }^{14}$ H. Haug and S. W. Koch, Quantum Theory of the Optical and Electronic Properties of Semiconductors, Third Edition (World Scientific, Singapore 1994).

${ }^{15}$ C. Comte and G. Mahler, Phys. Rev. B 34, 7164 (1986).

16 T. Iida, Y. Hasegawa, H. Higashimura and M. Aihara, Phys. Rev. B 47, 9328 (1993).

${ }^{17}$ H. Chu and Y. C. Chang, Phys. Rev. B 54, 5020 (1996).

${ }^{18}$ V. M. Galitskii, S. P. Goreslavskii and V. F. Elesin, Sov. Phys. JETP 30, 117 (1979).

${ }^{19}$ P. Nozières and S. Schmitt-Rink, J. Low Temp. Phys. 59, 195 (1985).

${ }^{20}$ R. Zimmermann, Phys. Stat. Sol. B 76, 191 (1976).

${ }^{21}$ P. Ring and P. Schuck, The Nuclear Many-Body Problem (Springer, New York, 1980).

FIG. 1. The mean interparticle distance $r_{\mathrm{s}}=(4 \pi n / 3)^{-1 / 3}$ as a function of pump-light frequency, $\omega_{\mathrm{L}}-E_{g}$, where $n$ is the electron-hole pair density. The interaction energies between particles and pump light $\lambda$ are chosen (top to bottom): 0.001, 0.1, $0.3,1.0$. The open and closed circles represent the point at which the pump-probe spectra are calculated for $\lambda=0.001$ and $\lambda=0.3$, respectively.

FIG. 2. The effective gap $G_{\text {eff }}$ as a function of the pump frequency $\omega_{\mathrm{L}}$. The interaction energies between particles and pump light $\lambda$ are chosen (bottom to top): $0.001,0.1,0.3,1.0$.

FIG. 3. The pump-probe spectrum in the high-density state outside the bistability region $\left(\omega-E_{g}=-0.5, r_{\mathrm{s}}=0.87\right)$. Here the interaction energy between particles and pump light is chosen as $\lambda=0.001$. (a) is given by the present theory, and (b) is given by the BCS-like mean field theory.

FIG. 4. The pump-probe spectrum in the low-density state outside the bistability region $\left(\omega-E_{g}=-4.0, r_{\mathrm{s}}=140\right)$. The interaction energy between particles and pump light is $\lambda=0.001$. (a) is given by the present theory, and (b) is given by the BCS-like mean field theory.

FIG. 5. The pump-probe spectrum for the states inside the bistability region $\left(\omega-E_{g}=-2.0\right)$. Here the interaction energy between particles and pump light is chosen as $\lambda=0.001$. (a) and (b) are the spectra for low-density state $\left(r_{\mathrm{s}}=86\right)$ calculated by the present theory and BCS-like mean field theory, respectively. (c) and (d) are the results for high-density state $\left(r_{\mathrm{s}}=1.0\right)$ given by the present theory and BCS-like mean field theory, respectively.

FIG. 6. The pump-probe spectrum for $\lambda=0.1,0.2,0.3$, in which the pump-light frequency is chosen as $\omega_{\mathrm{L}}-E_{g}=-0.5$. In this choice of $\omega_{\mathrm{L}}-E_{g}$, the dimensionless mean interparticle distance $\left(r_{\mathrm{s}}=0.87\right)$ is almost independent on $\lambda$ as shown in Fig. 1. 
FIG. 7. The pump-probe spectrum for $\omega-E_{g}=-1.8$, in which the dimensionless mean interparticle distance is $r_{\mathrm{s}}=1.0$. The interaction energy between particles and the pump-light is $\lambda=0.3$, in which resonatorless optical bistability disappears. (a) is given by the present theory, and (b) is given by the BCS-like mean field theory.

FIG. 8. The pump-probe spectrum for $\omega-E_{g}=-2.75$, in which the dimensionless mean interparticle distance is $r_{\mathrm{s}}=1.4$. The interaction energy between particles and the pump-light is $\lambda=0.3$, in which resonatorless optical bistability disappears. (a) is given by the present theory, and (b) is given by the BCS-like mean field theory.

FIG. 9. The pump-probe spectrum for $\omega-E_{g}=-2.9$, in which the dimensionless mean interparticle distance is $r_{\mathrm{s}}=1.8$. The interaction energy between particles and the pump-light is $\lambda=0.3$, in which resonatorless optical bistability disappears. (a) is given by the present theory, and (b) is given by the BCS-like mean field theory.

FIG. 10. The pump-probe spectrum for $\omega-E_{g}=-3.2$, in which the dimensionless mean interparticle distance is $r_{\mathrm{s}}=2.3$. The interaction energy between particles and the pump-light is $\lambda=0.3$, in which resonatorless optical bistability disappears. (a) is given by the present theory, and (b) is given by the BCS-like mean field theory. 







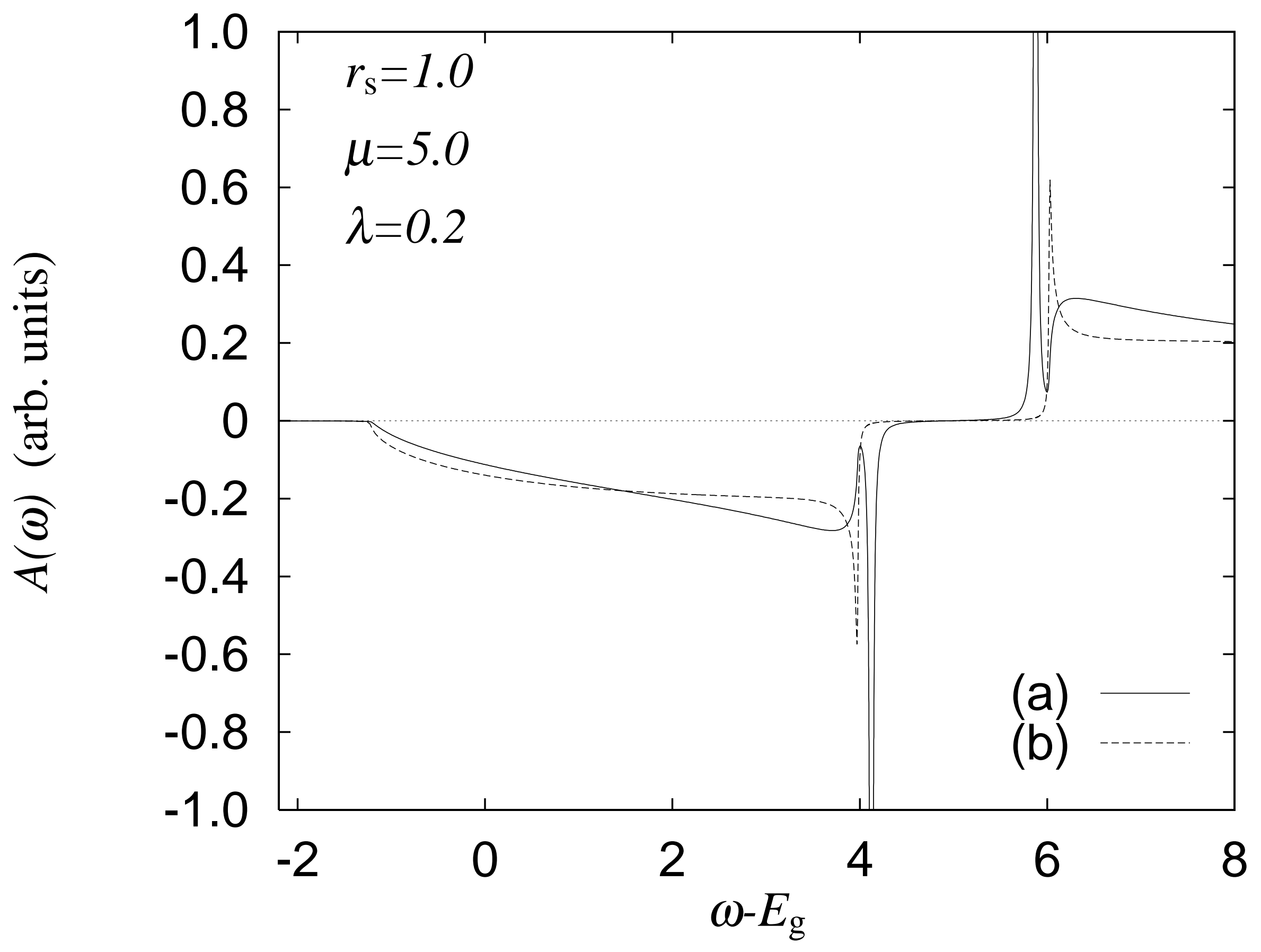




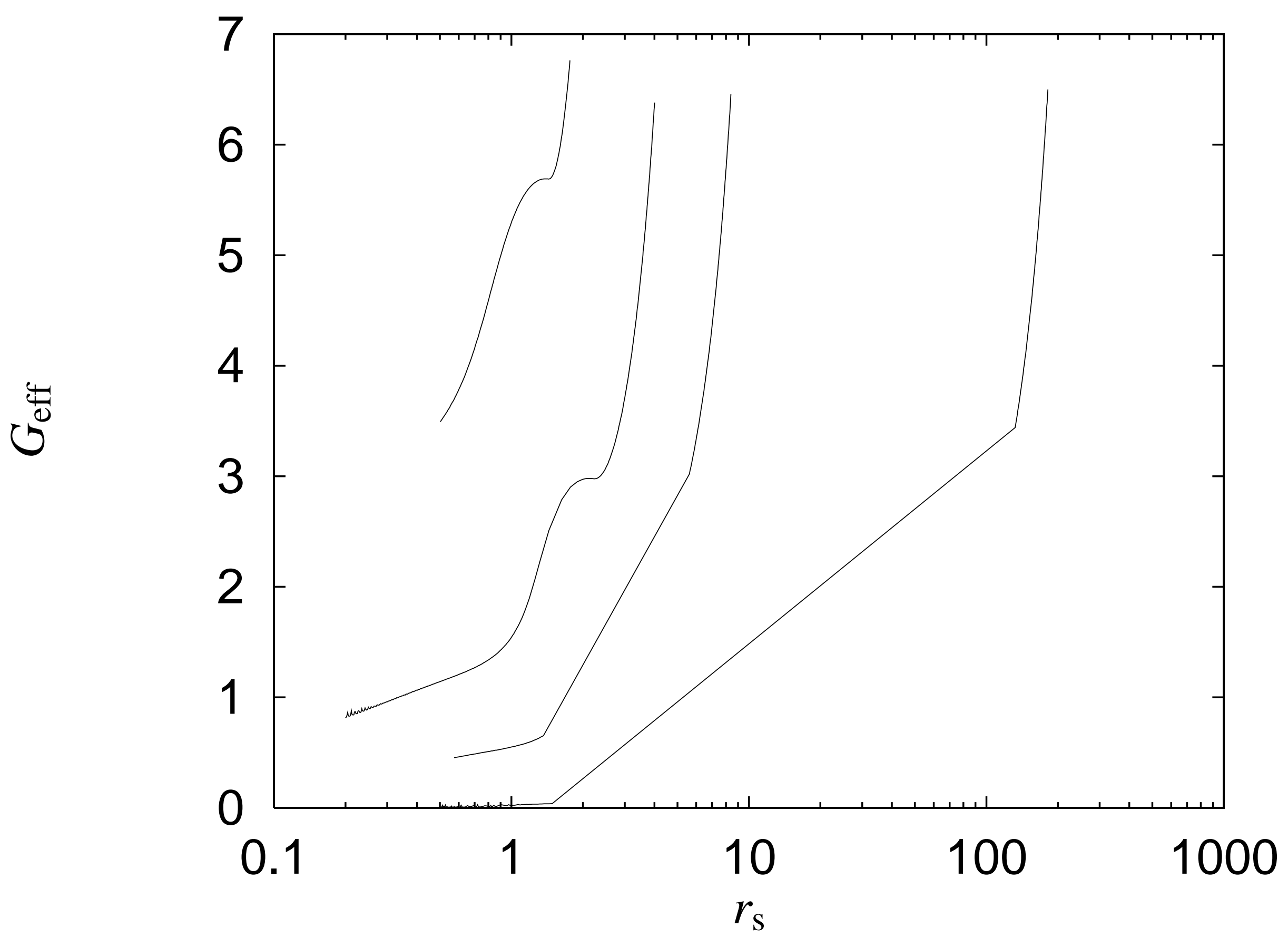




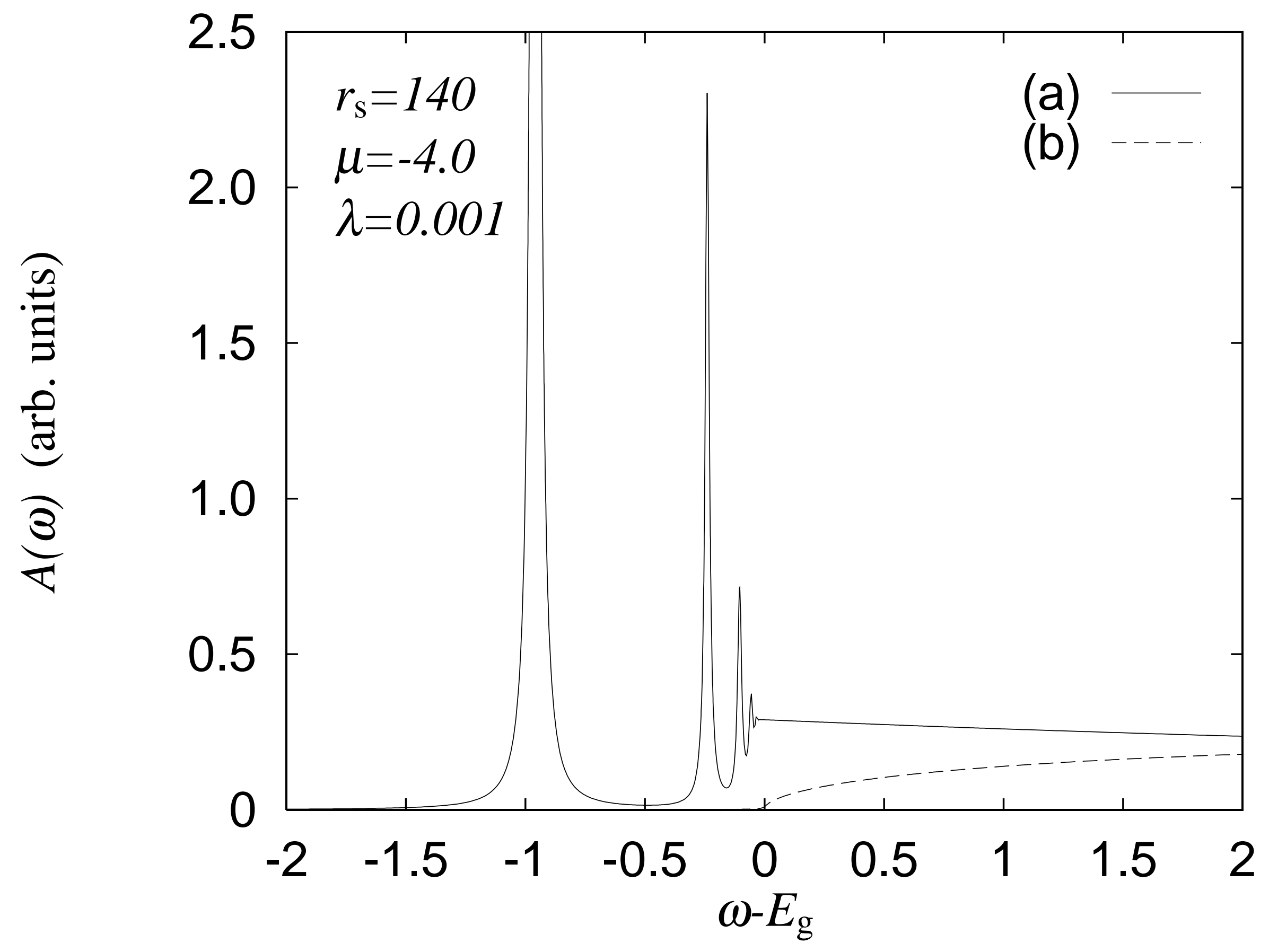




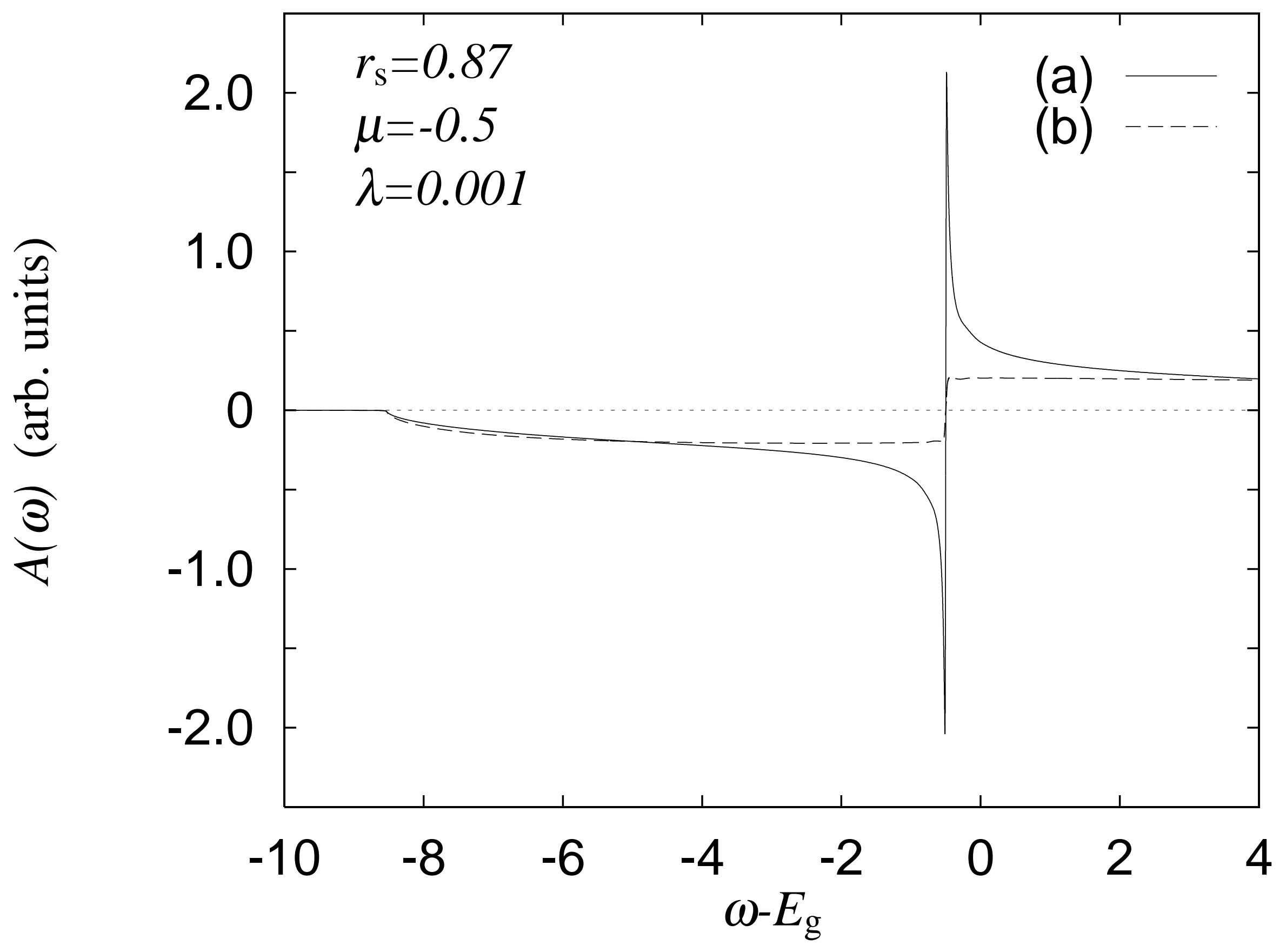




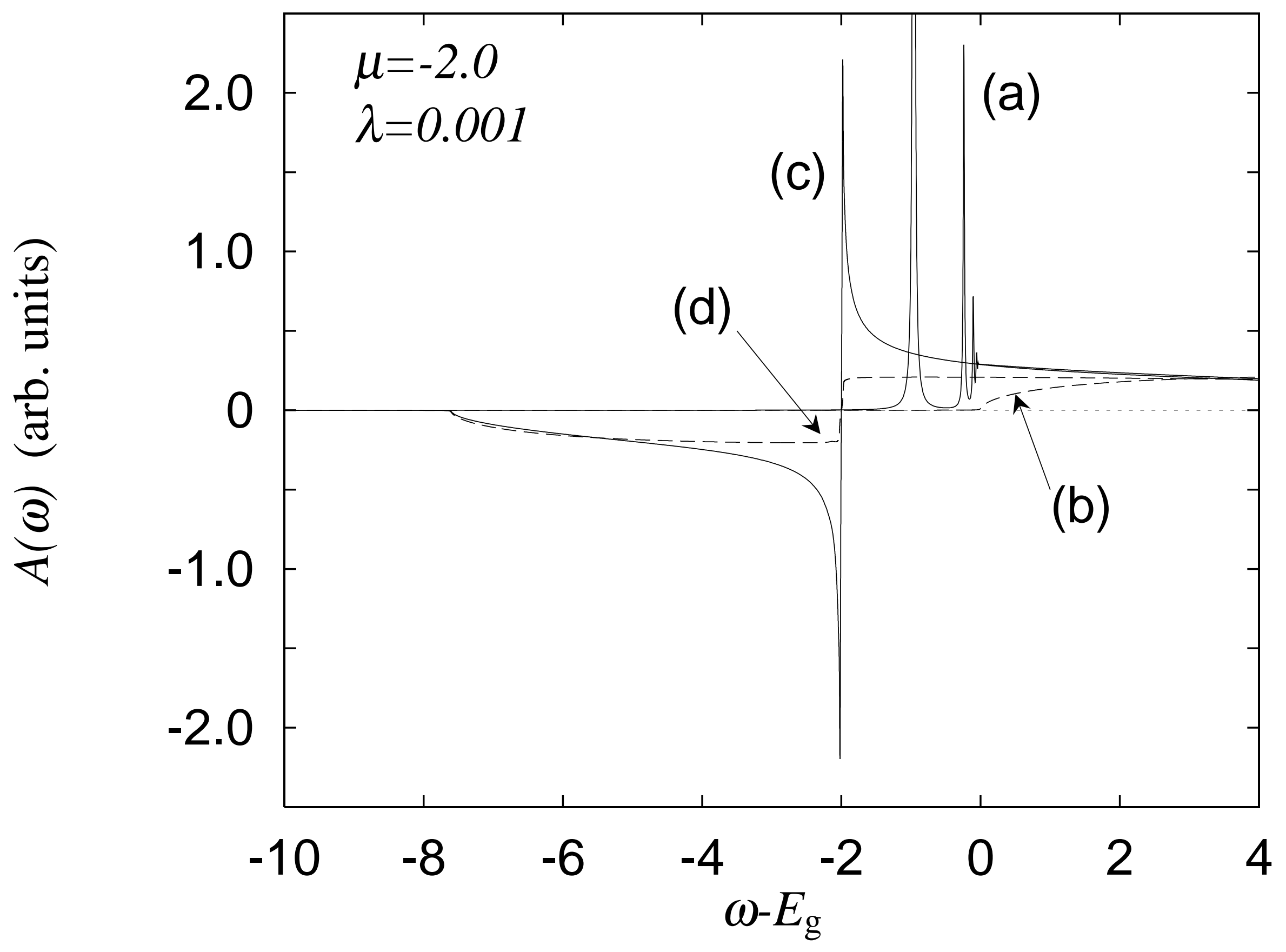




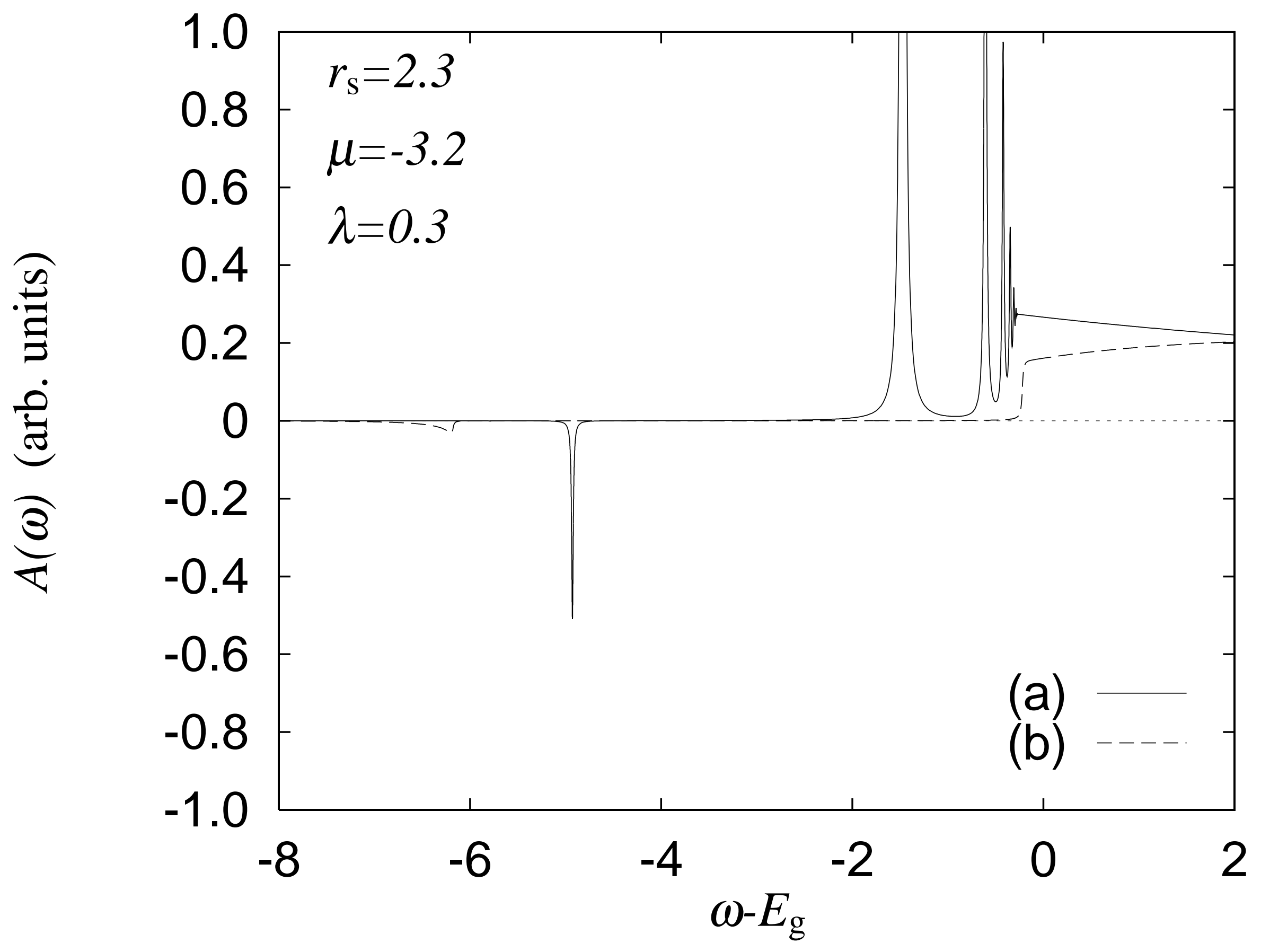




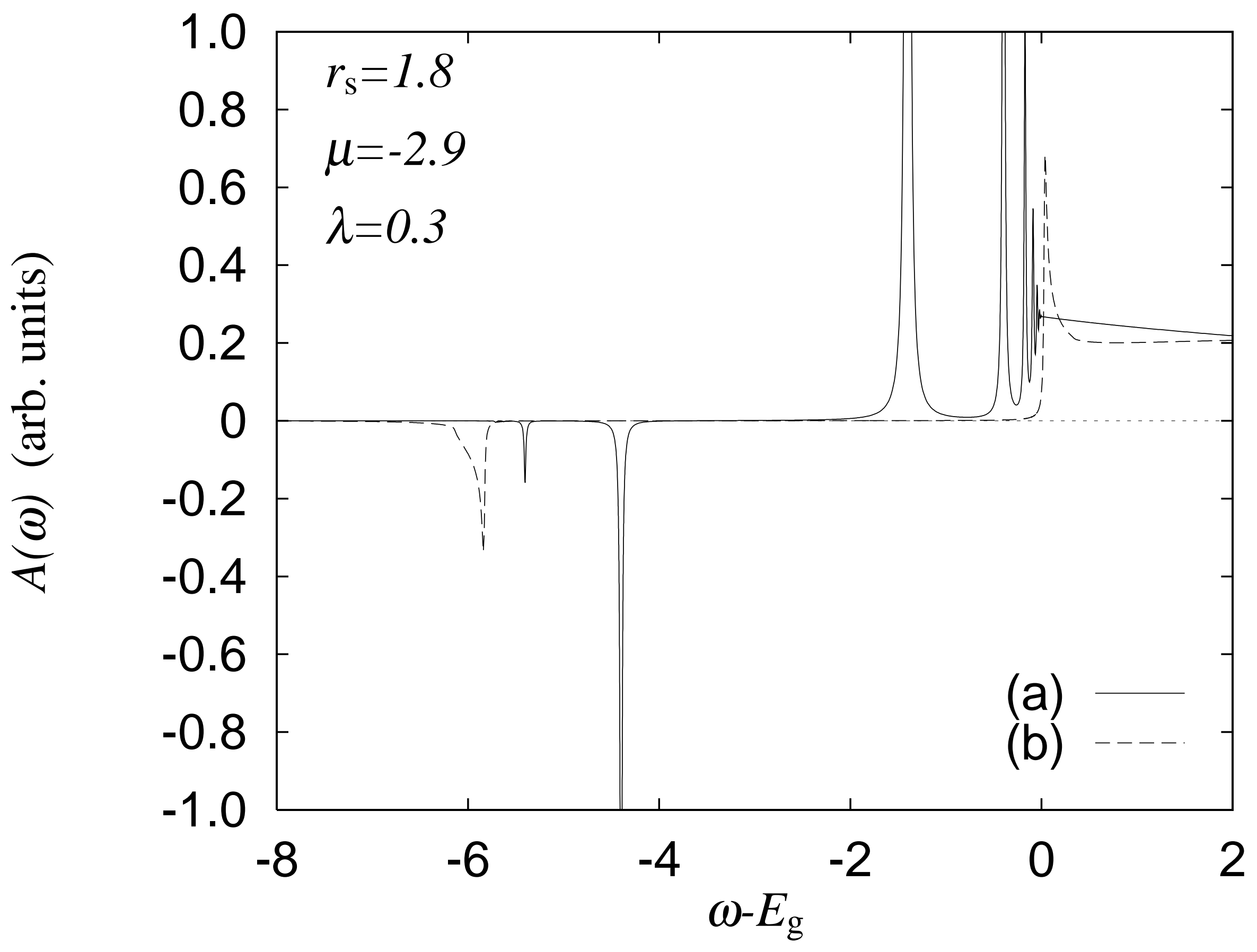




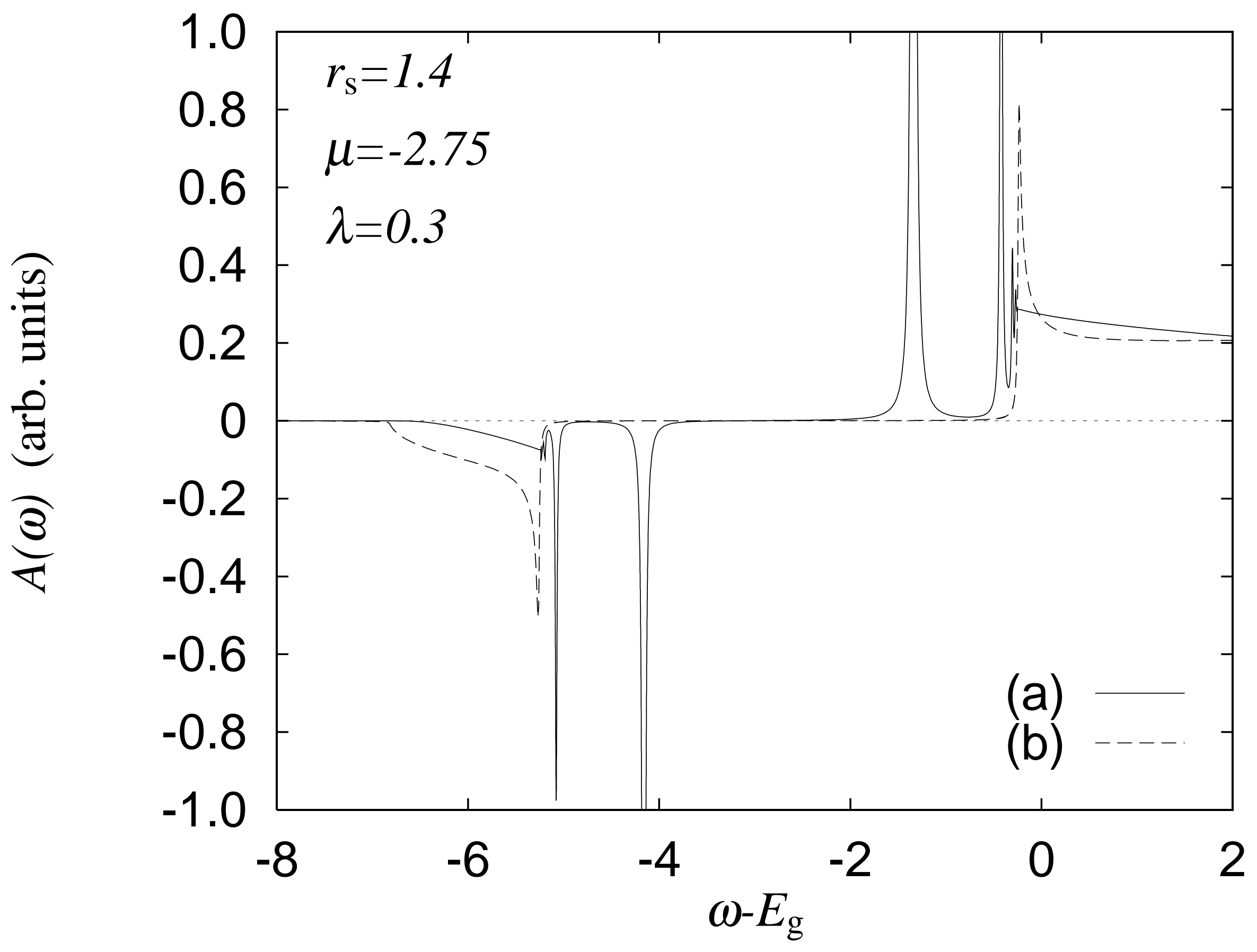




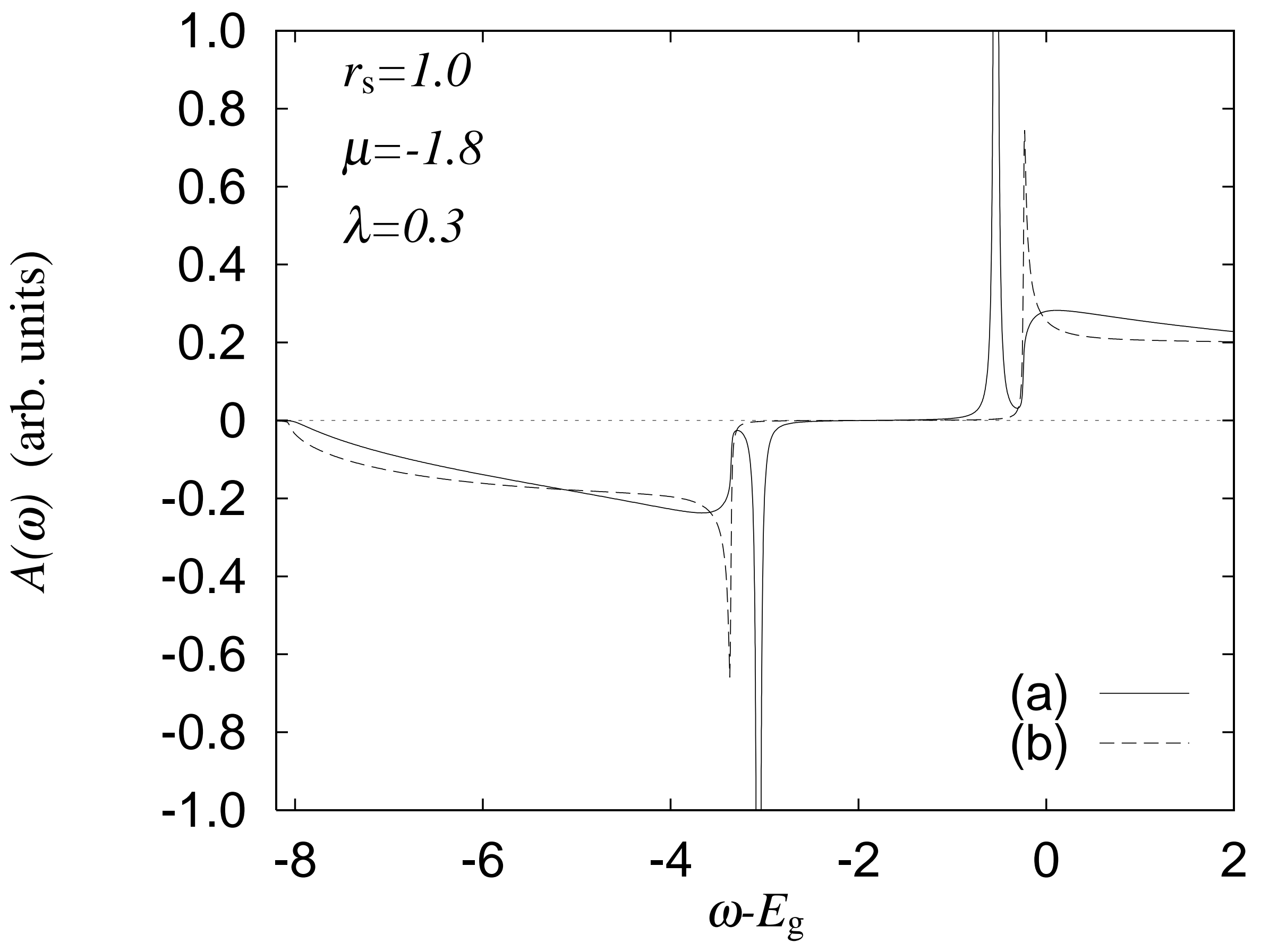

